\title{
Clinical Study the Relationship between High-TSH Degree and Changes of LDL-C Level in Pregnant Patients' Serum
}

\author{
Xu Ke* \\ Department of Endocrinology, First People's Hospital of Yancheng City, China
}

Received: January 17, 2018; Published: January 29, 2018

*Corresponding author: Xu Ke, Department of Endocrinology, First People’s Hospital of Yancheng City, Jiangsu Province, China; Email: ozawalanga@126.com

\section{Abstract}

Objective: It was to investigate the relationship between high-TSH degree and changes of LDL-C level in pregnant patients' serum and take analysis of its influencing effects.

Methods: All subjects were collected from the Yancheng First People's Hospital from January 2014 to December 2015, who was diagnosed by the Endocrinology Department. Then it analyzed their biochemical indicators and blood liquid parameters in their serum. Additionally, we investigated their epidemiological data, including the disease history and genes. At last it took regression analysis of its relevance.

Results: High-TSH degree and changes of LDL-C level are risk factors leading to hypertension, cardiovascular symptoms, symptoms of digestive tract, pregnancy ultrasound indicating small-sized fetus and they are related to one another $(\mathrm{P}<0.05)$; Whereas it has nothing to do with gestational diabetes mellitus, preterm birth history and autoimmune diseases. In the whole, high-TSH degree group, the high cholesterol could cause premature birth, elevated blood sugar, high blood pressure prenatal, postpartum infection and preeclampsia $(\mathrm{P}<0.05)$

Conclusion: These risk factors of high-TSH degree and changes of LDL-C level in serum could affect the health of pregnant, maternal and fetal, which should be taken to manage it so as to improve the perinatal health.

Keywords: High-TSH; LDL-C level; Pregnant patients

\section{Introduction}

Perinatal health care is a kind of system science which is used to survey embryonic development, fetal physiology and pathology as well as the diagnosis and prevention of illnesses of new born babies and pregnant woman $[1,2]$. How to control their endocrine hormone levels during perinatal period is the new driving direction of studying the early clinical prevention in high-risk pregnancy, among which TSH (Thyrotropin, thyroid stimulating hormone,TSH) coming from pituitary is a kind of hormone promoting the growth and function of thyroid, but is also one of the fundamental risky factors to high-risk pregnancy. TSH helps thyroid hormone release and T4, T3 synthesize $[3,4]$. Meanwhile, thyroid hormone regulates the lipid metabolism. The disorder of thyroxin may result in the changes of various liquid metabolisms [5-7]. Among patients with the function of sub-clinical thyroid and thyroid reducing, atherosclerosis accelerated by hyperlipidemia and the increase in the danger of arterial disease, like hypertensive disorders in pregnancy often come together. Obstetric bleeding, infection and convulsions often come with them. Thus, these are the main reasons causing death of maternal and perinatal women as well as infants. In order to appropriately control the thyroid hormone levels in a way to ensure the safety of pregnant women and infants so as to get prenatal and postnatal care done well, this study comprehensively analyzed such indicators as LDL-C level [8,9] and the result of postpartum of high-TSH pregnant having more than 12 gestational weeks. The details of case report are as follows.

\section{Materials and Methods}

\section{Subjects}

All the involved pregnant patients were diagnosed in the Yancheng First People's Hospital from January 2014 to December 2015, who was diagnosed by the Endocrinology Department. Their ages were from 21 to 43 , at average of $27.32 \pm 3.40$. The gestational time was above 12 weeks. But those having less than 12 gestational weeks were not involved in this study. According the difference of 
the pregnant patients' TSH degree, all 270 subjects were divided into three groups. In the control group, there were 127 cases with normal TSH degree during pregnancy. Ages vary from 20 to 41, $29.04 \pm 4.40$ on average. Their TSH serum levels were under 4.3 mIU/L. In the high-TSH group, it was consists of 143 cases. Their ages vary form 24 to 44 , with average ages of $29.53 \pm 4.80$. When their TSH degree was above $4.3 \mathrm{mIU} / \mathrm{L}$, they were considered as highTSH group. All these subjects were tested in pregnancy-induced hypertension and gestational diabetes mellitus, nephropathy and hypothyroidism and other diseases affecting blood liquid metabolism. The diagnosis standards were based on the obstetric diagnostic guild-line of The International Federation of Gynaecology and Obstetrics (FIGO). There are no statistic differences between normal control and high-TSH pregnant in age construction and gestational weeks. All materials involved are matched and tested.

\section{Survey Methods}

Patients' information was analyzed retrospectively and we designed special form for this study. Two trained professional and technical staffs have access to the patient's medical records. Data that need attention were included age, body weight, blood pressure, blood liquids, medical history and genetic history. Furthermore, the patient's epidemiological datum was obtained by telephone following-up for one year.

\section{Serological Analysis}

Venous blood sample collection schedule was based on weekly pregnancy time collected once three weeks. $3 \mathrm{ml}$ venous blood was collected in the morning before eating. Then separated the serum under $3500 \mathrm{rpm} / \mathrm{min}$ centrifuged at $4^{\mathrm{L}}$, which were stored under$20^{\mathrm{L}}$ for the next test. The test of serum (indicator including FT3, FT4 and TSH) was taken by Yancheng People 's Hospital Central Testing Laboratory. All tests were used the Chemiluminescence immunoassay methods. And the Automatic Chemiluminescence Analyzer was purchased from Jiangsu Ze Cheng Biotechnology Co., Ltd. (Model: CIA1200) (China). The detection reagent using for TC and LDL-C tesing was purchased from Sichuan Bayer Company

Table 1: Analysis of the difference of the clinical symptoms in pregnancy with high-TSH group between low and high LDL-C level subgroups.

\begin{tabular}{|c|c|c|c|c|c|c|c|}
\hline \multirow{2}{*}{ Clinical symptoms } & \multirow{2}{*}{ number } & \multicolumn{2}{|c|}{ High-TSH group } & \multicolumn{2}{|c|}{ Normal TSH group } & \multirow{2}{*}{$\mathbf{X}^{2}$} & \multirow{2}{*}{$\mathbf{P}$} \\
\hline & & Low LDL-C level & high LDL-C level & Low LDL-C level & high LDL-C level & & \\
\hline Pregnancy-induced hypertension & 21 & 4 & 16 & 0 & 1 & 12.61 & $\mathrm{p}<0.01$ \\
\hline Gestational diabetes mellitus & 12 & 3 & 4 & 2 & 3 & 0.22 & $\mathrm{p}>0.05$ \\
\hline Cardiovascular symptoms & 48 & 11 & 30 & 3 & 4 & 17.00 & $\mathrm{p}<0.01$ \\
\hline Digestive tract symptom & 64 & 12 & 22 & 14 & 16 & 1.13 & $\mathrm{p}>0.05$ \\
\hline $\begin{array}{l}\text { Pregnancy Ultrasound indicating } \\
\text { small fetus }\end{array}$ & 76 & 16 & 43 & 8 & 9 & 46.42 & $\mathrm{p}<0.01$ \\
\hline $\begin{array}{l}\text { Pregnant women with abnormal } \\
\text { body weight }\end{array}$ & 83 & 18 & 38 & 12 & 15 & 20.27 & $\mathrm{p}<0.01$ \\
\hline Personal or family history & 17 & 4 & 5 & 5 & 3 & 0.12 & $\mathrm{p}>0.05$ \\
\hline $\begin{array}{l}\text { The history of premature birth or } \\
\text { spontaneous abortion }\end{array}$ & 14 & 4 & 3 & 3 & 4 & 0.00 & $p>0.05$ \\
\hline Autoimmune disease & 11 & 3 & 3 & 3 & 2 & 0.18 & $\mathrm{p}>0.05$ \\
\hline
\end{tabular}

(Cat.No.2016071798). The inter variation and intra variation of the reagents in the experiment for quality-testing should be under $5 \%$.

\section{Statistical Analysis}

Statistical analyses were performed using IBM SPSS version 20.0 statistical software (IBM Corporation, Armonk, NY, USA). Datum were expressed as means and standard deviations. Analysis of variance (ANOVA) was used to compare the epidemiological data in each group. The paired test method was used to analyze the difference in the level changes of LDL-C. Additionally, Fisher's least-significant-difference test was used to compare the means between the groups. Values of $\mathrm{P}<0.05$ were considered statistically significant.

\section{Results}

\section{General Information}

It confirmed that high-TSH degree had a clear correlation with LDL-C level changes by laboratory tests serum, pregnant patients physical examination and epidemiological investigation. And importantly, both of them were high risk factors for the patients healthy. Furthermore, in this study, we defined the threshold value for these indicators as that TSH above $4.3 \mathrm{mIU} / \mathrm{L}$ and DL-C above $3.6 \mathrm{mmol} / \mathrm{L}$ in the pregnant patients' serum, which were approved by the by obstetric professional committee from Yanchang First People's Hospital. Additionally, the survey results on low density cholesterol threshold, along with the clinical performances of the 143 high TSH and 127 normal TSH which were shown in Table 1. As for the complications of high-TSH, it included pregnancy hypertension, cardiovascular symptoms, digestive tract system symptoms and small-sized fetus indicated by ultrasound, and they are related to each other $(\mathrm{P}<0.05)$. Whereas it had nothing to do with gestational diabetes mellitus, preterm birth history and autoimmune diseases. All induced the conclusion that high-TSH degree was related to the the growth of the fetus and vascular physiological changes of pregnant women, which should be paid high attention to and taken action to prevent. 


\section{LDL-C level of the Median}

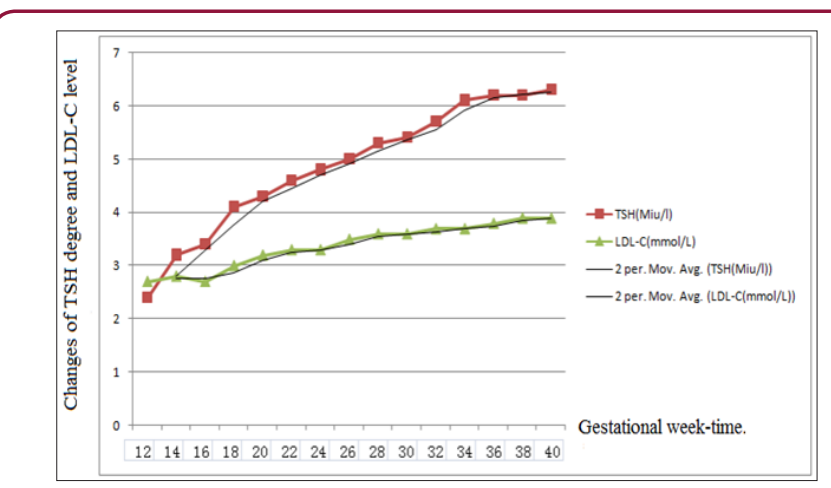

Figure 1: The changes in TSH degree and LDL-C level at different gestational week-time.

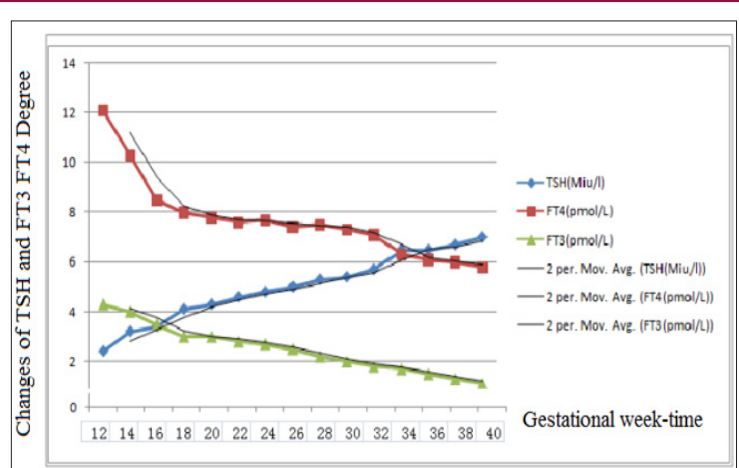

Figure 2: The changes in TSH and FT3 FT4 degree at different gestational week-time.

High-TSH interfered with the LDL-C level of the median. The dynamic change trend was analyzed during different gestational week-time by testing low density cholesterol level in the highTSH group. The finding indicated that TSH increased significantly with the increase of the gestational week-time. After analyzing the average trend, it had no sign to decrease. When analyzed the changes of LDL-C in the same pregnancy week time, it suggested that the increase of week-time in TSH begin from the forth week, and then it had an obvious increase. However, the slope of the growth trend of LDL-C level was not as high as the high-TSH degree, which might be affected by endocrine regulations of various hormone levels (Figures $1 \& 2$ ).

\section{Comparison of Postnatal Outcomes}

In this detection schedule, it was just tested during total pregnancy period. All patients were grouped again by the nonsequential TG level, according to the pregnant specificity. The $95 \%$ reliable data of pregnant TG should be taken as normal according to the value of T4. The 143 high-TSH patients were red vided into low blood liquid group (31) and high blood liquid group (112). Then it compared the postnatal outcomes both of pregnant mothers and children. There were significant differences in the premature delivery, prenatal hyperglycemia increasing, prenatal hypertension increasing and postpartum infection $(\mathrm{P}<0.05)$ between low and high blood liquid groups. High level of blood liquid could be a high risk for the high-TSH degree pregnant patients. But, it had no difference in the premature rupture of membranes, postpartum hemorrhage, fetal distress and placental abruption $(\mathrm{P}>0.05)$ (Table2).

Table 2: Control Study of high-TSH blood liquid group with normal TSH degree.

\begin{tabular}{|c|c|c|}
\hline Group & $\begin{array}{c}\text { Low blood liquid } \\
\text { group }\end{array}$ & $\begin{array}{c}\text { High blood liquid } \\
\text { group }\end{array}$ \\
\hline Number & 31 & 112 \\
\hline Premature delivery & 9 & $57 \#$ \\
\hline Increased blood sugar & 11 & $66 \#$ \\
\hline $\begin{array}{c}\text { Increased blood } \\
\text { pressure }\end{array}$ & 14 & $89 \&$ \\
\hline $\begin{array}{c}\text { Premature rupture of } \\
\text { membranes }\end{array}$ & 2 & 4 \\
\hline $\begin{array}{c}\text { Postpartum } \\
\text { hemorrhage }\end{array}$ & 3 & 4 \\
\hline Cesarean section & 3 & 6 \\
\hline Fetal distress & 1 & 3 \\
\hline Placental abruption & 2 & $32 \#$ \\
\hline Puerperal infection & 3 & 2 \\
\hline $\begin{array}{c}\text { Gestational } \\
\text { hypertension in later } \\
\text { life }\end{array}$ & 2 & 16 \\
\hline Abnormal liver function & 7 & 7 \\
\hline
\end{tabular}

Note: \# when compared with low blood liquid group, there were statistic differences $(\mathrm{P}<0.05)$; \& compared with low blood liquid group, there were notable statistic differences.

\section{Relationship Between TSH With FT3/4 FT4 in High Blood Lipids Group}

It was to understand the direct relationship between high TSH and FT3/4 in their serum. It took tracking analysis for the high-TSH group patients from 12 to 40 weeks by biochemical testing, whose results were listed in Table 2. Along with the rise of TSH degree, FT3 and FT4 showed a decrease, even FT4 decreased greatly during 12-18 weeks. Thus, it needed medicine to prevent this downward trend.

\section{Discussion}

Perinatal health care is an important part of perinatal medicine, whose aim is to decline the death rate of perinatal children and the incidence rate of pregnant women. When it comes to perinatal medicine, the scientist pays more and more importance on secretion regulation because women in pregnant period greatly influence the growth of fetus. TSH plays an important role in the maternity period. Clinical and non-clinical hypothyroidism as well as perinatal health care has been hotly discussed in recent years [35]. The high-TSH degree further influences the normal metabolism of blood liquids, forming endocrine systemic diseases. This study analyzes screening indications for thyroid function retrospectively, preliminarily discusses the basic strategies of thyroid function screening, and sets up some methods to prevent clinical risk factors for TSH and LDL-C so as to find the relationship between 
hypothyroidism, non-hypothyroidism and pregnant women with unusual pregnancy.

The study from the Third Military Medical University indicates thyroid dysfunction of women of childbearing age has an impact on the process and results of pregnancy [10]. Clinical hypothyroidism and sub hypothyroidism could lead to infertility. It is also related to spontaneous abortion, hypertensive disorder complicating pregnancy, premature labor, placental abruption, fetal distress and low birth weight infants. At the same time, it may cause new born babies with low intelligence. This result is consistent with the research result. Dr. Li Jianan [11] found that blood liquid index of pregnant women is higher in the late pregnancy than that in postpartum period by studying the changes of blood liquid of normal pregnant women in late pregnant period and postpartum period. It is obviously higher than the non pregnant group. The difference makes sense statically. However, researcher Chen Shuo [10] found that the increase in blood liquid of the pregnant women is quite normal in that period when the changes in blood liquid of pregnant women are involved. It has something to do with estrogen levels. The increase in blood liquid of pregnant women does not show illness because it is normal that blood liquid decreases in the postpartum period.

Study from the First Hospital of Peking University [12] showed that thyroid function screening indications and sub clinical hypothyroidism have an impact on the pregnant results among 548 cases in pregnancy. FT4 with TSH among 111 showed a drop. This trend and the sub clinical hypothyroidism are important in checking. Many hormone levels take place when pregnancy is involved, which clearly influences liquid and apolipoprotein metabolism. Estrogen makes LDL-C decline, while progesterone makes TG drop, LDL-C increase. The increase of LDL-C leads to arteriosclerosis. The incidence rate of pregnant sub clinical hypothyroidism worldwide is $2.2 \%$, but it is $5.32 \%$ in China, which is higher than that in the world. But now people at home and abroad do not reach an agreement on the strategies of screening of thyroid function during pregnancy $[13,14]$.

There is not a standard on clinical reference for thyroid function during pregnancy in the world, neither is sub clinical hypothyroidism $[15,16]$. The result of the relationship between sub clinical hypothyroidism and adverse pregnancy outcome is uncertain, making it hard to get a standard in drug intervention time, drug intervention dose, the effect achieved after intervention. This research committed by committee of the First People's Hospital in Yanchng is properly discussed and gets an effective method by retrospective analysis. It studies clinical risky factors and further analyzes the risk factors for adverse reactions in lowdensity cholesterol in serum by choosing TSH and combining FT3 and FT4 in a way to find the proper medicine using time. Studying drug intervention is still important for our hospital.

\section{References}

1. (2013) Global, regional, and national age-sex specific all-cause and cause-specific mortality for 240 causes of death, 1990-2013: a systematic analysis for the Global Burden of Disease Study 2013. Lancet 385: 117171.

2. Reiter RJ, Tan DX, Korkmaz A, Rosales-Corral SA (2014) Melatonin and stable circadian rhythms optimize maternal, placental and fetal physiology. Hum Reprod Update 20(2): 293-307.

3. Merck Manual of Diagnosis and Therapy, Thyroid gland disorders.

4. (2006) the American Heritage Dictionary of the English Language, $\left(4^{\text {th }}\right.$ Edn) Houghton Mifflin Company.

5. Beck-Peccoz P, Persani L (1994) Variable biological activity of thyroidstimulating hormone. Eur J Endocrinol 131(4): 331-340.

6. Sergi I, Papandreou MJ, Medri G, Canonne C, Verrier B, et al. (1991) Immunoreactive and bioactive isoforms of human thyrotropin. Endocrinology 128(6): 3259-3268.

7. Duntas LH, Tsakalakos N, Grab-Duntas B, Kalarritou M, Papadodima E (2003) The use of recombinant human thyrotropin (Thyrogen) in the diagnosis and treatment of thyroid cancer. Hormones 2(3): 169-174.

8. Dashti M, Kulik W, Hoek F, Veerman EC, Peppelenbosch MP, et al. (2011) A phospholipidomic analysis of all defined human plasma lipoproteins. Sci Rep 1(139).

9. Dashty M, Motazacker MM, Levels J, De Vries M, Mahmoudi M, et al. (2014) Proteome of human plasma very low-density lipoprotein and low-density lipoprotein exhibits a link with coagulation and lipid metabolism. Thromb Haemost 111(3): 518-530.

10. Sun Xiao, Zhang Lei, Yang Huixia, Zhou Yingfang, Sun Weijie (2014) the screening indication of thyroid function of 548 cases and the effects of subclinical hypothyroidism on pregnancy outcomes. Chin J Clin Obstet Gynecol 15(1):45-50.

11. Cheng Shuo, Ma Wuxiang, Liu Guohua (2005) Analysis of Changes of Blood Lipid in Normal Pregnant Women during Pregnancy. The $10^{\text {th }}$ National Military Medical Laboratory Paper Proceedings.

12. Moncayo R, Moncayo H (2017) A post-publication analysis of the idealized upper reference value of $2.5 \mathrm{mIU} / \mathrm{L}$ for TSH: Time to support the thyroid axis with magnesium and iron especially in the setting of reproduction medicine. BBA Clin 7:115-119.

13. Wang S, Teng WP, Li JX, Wang WW, Shan ZY (2012) Effects of maternal subclinical hypothyroidism on obstetrical outcomes during early pregnancy. J Endocrinol Invest 35(3): 322-325.

14. Cai Y, Zhong L, Guan J, Guo R, Niu B, et al. (2017) Outcome of in vitro fertilization in women with subclinical hypothyroidism. Reprod Biol Endocrinol 15(1): 39.

15. Zhang Y, Wang H, Pan X, Teng W, Shan Z (2017) Patients with subclinical hypothyroidism before 20 weeks of pregnancy have a higher risk of miscarriage: A systematic review and meta-analysis. PLoS One 12(4): e0175708.

16. (2009) Reproductive Health and Research Publications: Making Pregnancy Safer. World Health Organization Regional Office for SouthEast Asia. 
(C) (i) This work is licensed under Creative

Submission Link: http://biomedres.us/submit-manuscript.php

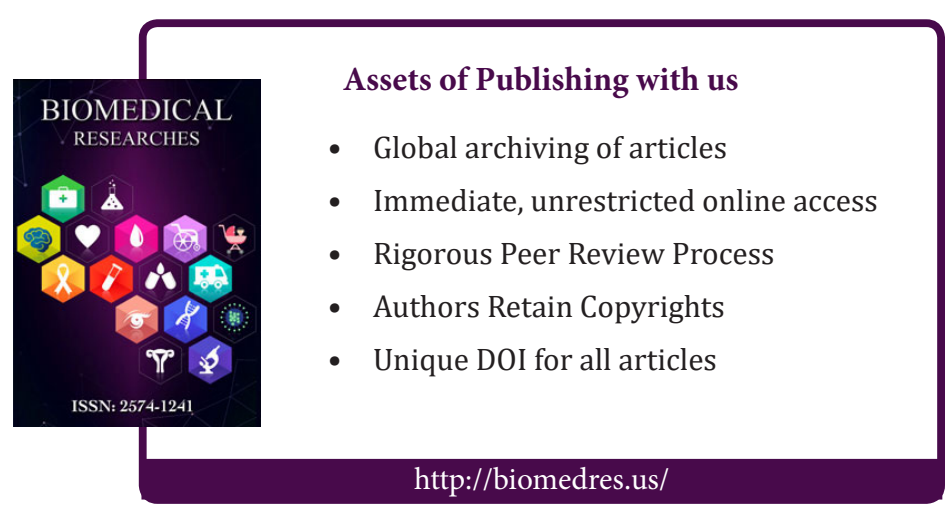

